\title{
Digit ratios have poor indicator value in a wild bird population
}

\author{
Suvi Ruuskanen • Samuli Helle • Markus Ahola • \\ Freya Adamczyck • Erich Möstl • Toni Laaksonen
}

Received: 2 August 2010 /Revised: 25 October 2010 /Accepted: 25 October 2010 /Published online: 12 November 2010

(C) The Author(s) 2010. This article is published with open access at Springerlink.com

\begin{abstract}
Early androgen exposure is known to have longlasting effects on phenotype, behaviour and even fitness, but difficulties in measuring the exposure hinders the study of its importance in evolutionary context. Digit ratios have been highlighted as a potential easy-to-measure indicator of early steroid exposure, as they have been suggested to reflect steroid, mainly testosterone levels during prenatal development. However, evidence for digit ratios reflecting early steroid levels is weak, as experimental studies, especially in wild populations, are scarce. We studied the association between maternally derived yolk androgens and digit ratios (2D:4D, 2D:3D and 3D:4D) using both correlative data and a rather high level of experimental elevation of yolk androgens in a passerine bird, the pied flycatcher (Ficedula hypoleuca). We also examined whether digit ratios have indicator value in an evolutionary context by studying correlations between digit ratios and reproductive traits, secondary sexual traits and exploratory behaviour. We did not find any association between digit
\end{abstract}

Communicated by J. Graves

S. Ruuskanen $(\bowtie) \cdot S$. Helle $\cdot$ M. Ahola $\cdot$ T. Laaksonen

Section of Ecology, Department of Biology, University of Turku,

Turku, Finland

e-mail: suvi.ruuskanen@utu.fi

F. Adamczyck

Department of Animal Population Biology,

Netherlands Institute of Ecology (NIOO-KNAW),

Heteren, The Netherlands

E. Möstl

University of Veterinary Medicine,

Vienna, Austria

T. Laaksonen

Finnish Museum of Natural History, University of Helsinki, Helsinki, Finland ratios and yolk androgen level either in correlative or experimental data. Digit ratios were neither related to any of the reproductive and secondary sexual traits or exploratory behaviour measured. There was, however, a sex difference in 2D:3D and 3D:4D of adult birds (due to second and fourth digits being shorter in females), which was not apparent in fledglings or captivity-raised juveniles. This suggests that either the sex difference may develop as late as during the sexual maturation for breeding. These results indicate that, in this species, digit ratios are not reliable markers of maternally derived yolk androgen exposure and that they bear little relevance as correlates of the adaptive traits we measured.

Keywords 2D:4D · Bird · Fitness · Testosterone $\cdot$ Maternal effects $\cdot$ Sexual dimorphism

\section{Introduction}

Digit length ratios (especially the ratio of second to fourth digit length, 2D:4D) have been reported to correlate with several phenotypic traits and behaviours (reviewed by Manning et al. 2000; Manning 2002; McIntyre 2006, but see, e.g. Putz et al. 2004 for a critical review). It has been suggested that these correlations arise because both digit ratios and many phenotypic and behavioral traits reflect steroid exposure during early development (e.g. Clark and Galef 1995; Manning et al. 1998; Dufty et al. 2002; CohenBendahan et al. 2005; Groothuis et al. 2005; McIntyre 2006; Hönekopp et al. 2007; Garamszegi et al. 2008). Digit ratios have thus been suggested to be potential easy-tomeasure phenotypic biomarkers of early hormone exposure, which could facilitate the study of the evolutionary importance of early hormone exposure. 
The main problem with the digit ratio theory is that we do not currently know what digit ratios really reflect, as direct evidence for the role of early steroid exposure is very scarce and restricted to few captive model species. Birds and other egg-laying animals are excellent models for studying the potential association between early hormone exposure and digit ratios, as hormone levels inside the eggs can be manipulated (e.g. Groothuis and von Engelhardt 2005). Surprisingly, only two experimental studies have been carried out in egg-laying animals (androgens, Romano et al. 2005; estradiol, Saino et al. 2007; see Talarovičová et al. 2009 for an androgen-manipulation in rodents). The study by Romano et al. (2005) showed that in captive strains of pheasants (Phasianus colchicus), high androgen exposure potentially leads to higher 2D:3D (but not 2D:4D) ratios in females but not in males. Using the same species, Saino et al. (2007) showed that estradiol decreased 2D:4D ratio in males but had no effect on females. Indirect approach to this question has also been applied. Burley and Foster (2004) found that, in zebra finches (Taeniopygia guttata), digit ratios varied within the laying order in a similar manner as maternally derived yolk androgens, suggesting a potential association between the two. However, Forstmeier (2005) did not found any association between digit ratios and laying order in the same species. There is thus clearly a need for more experimental studies on steroid-related variation in digit ratios.

If digit ratios reflect the early hormone environment, an important additional question is whether they correlate with important fitness-related reproductive traits or behaviours. In the most widely studied species, humans, the results of the correlations between digit ratios and phenotypic traits (e.g. cognitive abilities, dominance, personality, vigour and fecundity) are highly controversial, perhaps due to numerous methods used to examine digit ratios (reviewed by Putz et al. 2004). Results of the few studies on birds are also indicative of relationships but yet far from conclusive. Activity, male song rate and mate choice of females have been found to correlate with digit ratios in captive zebra finches (Burley and Foster 2004; Forstmeier 2005). In house sparrows (Passer domesticus), digit ratios correlated with a sexually selected trait, male badge size (Navarro et al. 2007) and, in barn swallows, (Hirundo rustica) with tail length and body mass (Dreiss et al. 2008). However, in the collared flycatcher (Ficedula albicollis), song or plumage traits were not associated with digit ratios (Garamszegi et al. 2008). At present, it is thus not known whether digit ratios can be used as a correlate for important fitness-related reproductive traits, secondary sexual traits or behaviour in birds or any free-living vertebrates.

The main aim of this study was to test whether digit ratios serve as a phenotypic indicator for maternally derived early androgen exposure in a small passerine bird, the pied flycatcher (Ficedula hypoleuca). First, we studied the association between maternally derived yolk androgens and digit ratios using both correlative data and experimental manipulation. Second, we tested whether there is a sexdifference in digit ratios. Third, we studied whether digit ratios were associated with important fitness-related traits (laying date and clutch size in females and arrival date in males), secondary sexual traits of males [forehead patch size and plumage ultraviolet (UV) coloration] or individual behaviour (exploratory behaviour, activity, neophobia). The aim of these latter analyses was to examine whether digit ratios, regardless of what they might be reflecting, have any indicator value in an evolutionary context.

\section{Methods}

\section{Study species}

The pied flycatcher is a small (12-13g), migratory, insectivorous, hole-nesting passerine bird (Lundberg and Alatalo 1992). It breeds in large range over Europe and winters in western Africa. Pied flycatchers are singlebrooded, and the modal clutch size is five to six eggs. The pied flycatcher is an abundant species that easily accepts artificial nest boxes, and thus, it is a common model species in studies of avian ecology and evolution.

\section{Correlative data from the cross-fostering experiment}

\section{Field protocol}

The data was collected in a nest box breeding population of pied flycatchers in Turku, Finland $\left(60^{\circ} 26.055^{\prime} \mathrm{N}\right.$, $22^{\circ} 10.391^{\prime}$ E) in summer 2005. To record arrival date of the males, the surroundings of all nest boxes were routinely monitored daily from 1st of May until the end of May (the first male in the study area was seen 3rd of May). The contents of nest boxes were routinely monitored for nest initiation and egg laying every third day since the beginning of May. Pied flycatchers lay one egg per day, and we could thus detect each nest before the laying of the fourth egg. The laying date of the first egg could also be determined this way. The nests were visited on the day of laying the third egg, and the eggs were marked with small dots (non-toxic permanent marker). On the following day, the fourth, non-marked egg was collected and replaced with a dummy egg, to maintain the same incubation effort. Androgen content of the collected egg was subsequently used to estimate the average yolk androgen level of the clutch. Androgen levels may increase or decrease with laying order, but in most species studied so far, including the pied flycatcher, between-clutch variation in yolk 
androgen levels is higher than within-clutch variation (e.g. Reed and Vleck 2001; Groothuis and Schwabl 2002; Pilz et al. 2004; Tschirren et al. 2004; Michl et al. 2005; Tobler et al. 2007; T. Laaksonen, unpublished data). Even if within-clutch variation in hormone deposition would occur, the fourth egg would still represent the medium concentration of the whole clutch, as long as the pattern of withinclutch variation is linear (as found in many studied species with significant within-clutch variation, e.g. Reed and Vleck 2001; Groothuis and Schwabl 2002; Pilz et al. 2004; Tschirren et al. 2004). Furthermore, based on 24 clutches collected from the study population in 2004, the androgen levels in the fourth egg are strongly correlated with the clutch mean $\left(r_{\text {Spearman }}=0.85\right.$ for testosterone and $r_{\text {Spearman }}=0.78$ for androstenedione). The collected egg was weighed fresh to the nearest $0.01 \mathrm{~g}$ with a digital scale and thereafter frozen at $-20^{\circ} \mathrm{C}$. The concentrations of immunoreactive androstenedione and testosterone in the yolks were measured later in the lab (see below). Eggs from 96 nests were collected. As the eggs were collected before incubation, the androgen level we measured in the eggs is completely of maternal origin and not produced by the embryo. Nests were further monitored to record the final clutch size.

Full clutches of eggs were swapped between two (sometime three) nests with the same laying date in the middle of the incubation period (5 days after clutch completion) for the purposes of another experiment (Laaksonen and Lessells 2006; Laaksonen et al. 2010). Cross-fostering full clutches diminishes the resemblance of offspring and parents caused by common environment effects. The nests were visited daily at the time of the estimated hatching (after 14 days of incubation), to get an accurate estimate of hatching date. For the purposes of another experiment (Laaksonen et al. 2010), brood size was further standardised to six chicks 1 or 2 days after hatching; standardisation removes here the variation potentially caused by brood size. On day 5 post-hatching, chicks were ringed and blood sampled from the brachial vein for collecting DNA for molecular sexing (see details below). Digit ratio measurements from the chicks were taken at the age of 12 days, when the chicks were fully grown to and about to fledge in 2 days (see details of measurements below).

Female parents were captured at the end of the incubation period either by hand or using a swing-door trap when they returned to incubate. Male parents were captured with a swing-door trap 6-7 days after the chicks hatched. Digit ratio measurements were taken when birds were caught (see details of the measurements below). Altogether, 77 parent females and 68 males were measured. Forehead patch size and feather UV reflectance of the males was measured. The forehead patch of male pied flycatchers is a highly variable feature of the breeding plumage
(Lundberg and Alatalo 1992). Forehead patch size was measured from digital photographs taken in the field with a millimetre grid held next to, and at the same level and angle as the patch. The patch area $\left(\mathrm{mm}^{2}\right)$ was measured using Image-J freeware and manual selection of the patch boundaries. Female pied flycatchers choose their mate on the basis of the brightness of male coloration at UV wavelengths (Siitari et al. 2002), and this trait also affects the probability of the male losing paternity in his nest (Lehtonen et al. 2009). UV coloration was measured from the central tertial feather, which was collected from each individual. This feather is at the centre of the white wing patch, which is much larger in the breeding plumage of males than females. We measured UV coloration from this feather because white feathers typically have higher UV reflectance than dark feathers (Eaton and Lanyon 2003), and the middle tertial has a rigid structure, whereas the dark or brown body feathers on the back have a soft semi-plume structure. The UV chroma [proportion of reflectance in the UV spectrum, i.e. (reflectance at $320-400 \mathrm{~nm}$ )/(reflectance at $320-700 \mathrm{~nm}$ )] was measured in the lab with a spectrophotometer (Avantes 2048, Avantes DH-S light source; white standard WS-2).

\section{Androgen analysis}

For measuring the concentrations of androgens [testosterone ( $\mathrm{T}$ ) and androstenedione (A4)] in yolk, we used a method similar to that described in Hackl et al. (2003) and Bertin et al. (2008). To extract steroids, after thawing, each yolk was suspended in $1 \mathrm{ml}$ distilled water and $4 \mathrm{ml}$ methanol and vortexed twice for $30 \mathrm{~s}$. Samples were then stored overnight at $-20^{\circ} \mathrm{C}$ overnight to precipitate apolar lipids. After centrifugation $\left(-15^{\circ} \mathrm{C}, 2,500 \times g, 10 \mathrm{~min}\right)$, $100 \mu \mathrm{l}$ of the supernatant were diluted with $400 \mu \mathrm{l}$ assaybuffer (1:5). For enzyme immunoassays, we used $20 \mu \mathrm{l}$ of sample dilution (for full descriptions of antibodies and validation see Palme and Möstl 1994; Hirschenhauser et al. 1999; Möstl et al. 2001). Inter-assay variation was 9.9\% (low level pool) and 5.5\% (high level pool) for testosterone and $12.9 \%$ and $9.3 \%$ for androstenedione. Intra-assay variation was $7.9 \%$ for testosterone and $10.1 \%$ for androstenedione.

Androgen manipulation experiment

\section{Field and injection protocol}

The androgen manipulation experiment was conducted in summer and autumn 2008 in the same population of pied flycatchers as where the correlational data was collected. Full description of the field and injection protocol is found in Ruuskanen and Laaksonen (2010). Briefly, on the 
estimated day of clutch completion, each clutch was randomly assigned to either the androgen or the control treatment. All the eggs in a single clutch were treated the same way. In the androgen treatment (initial $N=31$ clutches), the eggs were injected with $7.5 \mathrm{ng}$ of testosterone (Fluka) and $53.1 \mathrm{ng}$ of androstenedione (Fluka) dissolved in $4 \mu$ l sesame oil. In the control treatment (initial $N=25$ clutches), the eggs were injected only with $4 \mu$ l of sesame oil. The amount of injected androgens was calculated using previous data on natural yolk androgen levels from 24 clutches in the same population (testosterone mean $\pm \mathrm{SD}$, $4.6 \pm 2.1 \mathrm{ng} /$ yolk; maximum, $12.1 \mathrm{ng} /$ yolk; androstenedione mean $\pm \mathrm{SD}, 35.6 \pm 17.0 \mathrm{ng} /$ yolk; maximum, $88.6 \mathrm{ng} /$ yolk; Laaksonen, unpublished data). The amount injected corresponds to the difference between the mean and maximum values of androgens per yolk, ensuring that the final yolk androgen level in the androgen-treated clutches was on average at the upper limit of the natural range. The injected amount of androgens corresponded to 3.57 and 3.12 times the SD in testosterone and androstenedione, respectively. Eggs with very high natural levels thus probably had values above the previously measured range; however, the distribution of high- and low-androgen-level eggs is very skewed (with most eggs having low levels of androgens and few high levels of androgens), which makes it likely that the androgen level is above the physiological range in a small number of eggs. We think that if any association between yolk androgen levels and digit ratios exists, using a relatively high level of elevation of androgen levels should reveal this association. The position of the yolk was visualised using a light source positioned beneath the egg. The surface of the egg was first cleaned with $95 \%$ ethanol, and a small hole was made using a disposable $27 \mathrm{G}$ needle. The oil vehicle was injected into the yolk using a $25-\mu 1$ Hamilton microliter syringe (702RN) and $26 \mathrm{G}$ needle. After the injection, the hole in the egg shell was sealed with a drop of tissue adhesive (Vet-Seal, B. Braun Medical, Switzerland). The eggs were returned to the nest immediately after the injections. The nests were checked on the following 2 days, and the seventh and eighth eggs, if present, were likewise injected, according to the clutch treatment. This method of manipulating yolk androgen levels has been successfully used and validated in previous studies (e.g. Tschirren et al. 2005; Ruuskanen et al. 2009; Ruuskanen and Laaksonen 2010).

The hatching success (mean \pm SD) was $70.3 \pm 18.1 \%$ for the control treatment group and $76.1 \pm 17.1 \%$ for the androgen treatment group. There was no significant difference between the groups using logistic regression analysis $\left(\chi^{2}=1.10, p=0.29\right)$. The hatching success (mean \pm SD) of non-injected clutches (nests with zero hatchlings excluded $)$ in this population is $90.5 \pm 15.4 \%(N=181$, data from years 2004 and 2006; Laaksonen T., unpublished data). The nests were monitored to record final clutch size and hatching date as a part of population monitoring. On the fifth day after hatching, the chicks were ringed, and blood samples were taken from the brachial vein to collect DNA for molecular sexing, which was carried out during the nestling period. Full description of the molecular sexing is found in Ruuskanen and Laaksonen (2010).

\section{Housing in the aviaries}

For a full description of the housing conditions, see Ruuskanen and Laaksonen (2010). Twelve days after hatching, two chicks - one male and one female-from each of 30 broods $[15$ androgen treated (AT) and 15 control nests $(\mathrm{CO})]$ were transferred to the aviaries. These 60 nestlings were arranged into ten new foster broods, with a brood size of six chicks. Siblings were placed in the same foster brood, with each foster brood including chicks from both treatments. Each brood was fed in the aviaries $(2.5 \times$ $3 \times 2.5 \mathrm{~m}$ in size) by two foster parents until it was certain that the young could forage independently (at an age of about 40 days), after which the foster parents were released. Two aviaries were thereafter connected, and the birds were kept in groups of 12 individuals (six males and six females, a roughly equal number of birds from both treatments) for the rest of the year. Digit ratio measurements were taken when birds were approximately 12 weeks old (see details below). The rearing of the birds in captivity and the following experiments were conducted under license from the Animal Experiment Committee of the State Provincial Office of Southern Finland (license number ESLH-200803693) and the Environmental Center of Southwestern Finland (license number LOS-2007-L-264-254).

\section{Behavioral measures (novel environment and novel object experiments)}

The protocols for these experiments were modified from Verbeek et al. (1994). For a full description of the experiments, see Ruuskanen and Laaksonen (2010). The experiments were conducted on approximately 12-week-old birds. Briefly, 1 day before the experiments, the birds were captured in the aviaries and placed in individual cages. Each bird was tested individually, and observations were made intermittently between 9 A.M. and 5 P.M.

The novel environment experiment was conducted to measure exploration and activity by individual birds in a novel environment. The experiment was conducted in an observation room $4 \times 2 \times 2.5 \mathrm{~m}$ in size, which contained five artificial wooden trees $1.5 \mathrm{~m}$ in height, with four $20-\mathrm{cm}$ long cylindrical branches. The birds were captured in their cages and transported in cloth bags to the experimental room, where they were immediately released, and their 
behaviour was observed for $15 \mathrm{~min}$. We recorded the time and duration of every flight or hop made by a bird. In contrast to the behaviour of great tits (Parus major; see e.g. Verbeek et al. 1994), only few birds visited all five trees; many did not explore the room at all during the 15-min period. Thus, we did not consider the latency to visit all trees a particularly informative response variable. Instead, we used a categorical variable, exploring/not exploring the room and the sum total of all hops and flights during the 15-min period (hereafter "activity in the novel environment experiment"). Median number of hops and flights (per $15 \mathrm{~min}$ ) was 2.0 ( $\min , 0$; max, 43).

The novel object experiment was conducted to measure the boldness of individuals with regard to novel objects (i.e. neophobia) and their activity in the presence of one. The novel object, a pink and gold toy rubber duck, was placed on a perch in the bird's home cage. The behaviour of the bird was recorded with a video camera for 3 min after the object was introduced. To quantify the bird's reaction, we measured latency to approach the object and activity of the bird (total number of hops and flights during $3 \mathrm{~min}$ ). Median number of hops and flights (per $3 \mathrm{~min}$ ) was 69.0 (min, 3; max, 134).

\section{Digit ratio measurements}

The same protocol was used in both 2005 and 2008 to avoid any variation in methodology. Toes were straightened and pressed gently against a glass sheet (with a millimetre grid for calibration on the side), which was attached to a digital camera (Nikon Coolpix 4500). The lengths of second, third and fourth digits were measured from the digital photos with a programme Image-J, using the millimetre grid as a scale in each photo. The measurement technique we used is found to correlate quite strongly with bone lengths (Leoni et al. 2008), and thus, estimates of digit ratios based on whole digits largely convey the same biological information as those based on exact bone lengths. The digital measurement method was chosen, as it likely yields the most accurate and repeatable measurements (see, e.g. Allaway et al. 2009). The same person measured all photos, and two independent measures (at separate time points) were made to calculate the repeatability of digit ratios. The repeatability of the measurements in 30 double measures in the correlational data was 0.97 for $2 \mathrm{D}: 4 \mathrm{D}\left(F_{29,30}=58.15, p<0.0001\right), 0.95$ for 2D:3D $\left(F_{29,30}=39.45, p<0.0001\right)$ and 0.94 for 3D:4D $\left(F_{29,30}=33.37, p<0.0001\right)$. The corresponding triple measurements from the androgen manipulation experiments were as follows: 0.79 for $2 \mathrm{D}: 4 \mathrm{D} \quad\left(F_{57,116}=12.18, p<\right.$ $0.001), 0.60$ for 2D:3D $\left(F_{57,116}=4.98, p<0.001\right)$ and 0.70 for 3D:4D $\left(F_{57,116}=8.07, p<0.001\right)$. Digit ratios from 58 birds from the androgen-manipulation experiment could be measured, and average of the three measurements was used in the analyses. Measures were taken only from the right foot, as for example sexual differences are known to be more pronounced in the right foot (e.g. Brown et al. 2002; Dreiss et al. 2008).

\section{Statistical analyses}

The association of yolk androgens (A4 and T) with the digit ratios of chicks was examined using general linear mixed models, where the nest of origin was fitted as a random factor to control for the within-brood resemblance of the digit ratios (Littell et al. 2006). The variance inflation factor between the concentrations of the two hormones was 1.47, suggesting no collinearity problem. To examine whether these yolk androgens had sex-dependent associations on chick digit ratios, their interactions with offspring sex were included in the models. Assumption of heterogeneous residual variance by age and sex was examined using Akaike's information criteria (AIC), and the residual variance structure having the lowest AIC value was chosen (Littell et al. 2006). In the model examining 2D:4D, the model assuming unequal residual variance among the sexes fitted the data better $\left(\Delta \mathrm{AIC}_{\mathrm{C}}=1.7\right)$ than the equal variance model. Therefore, the analysis of $2 \mathrm{D}: 4 \mathrm{D}$ also accounted for unequal residual variance among the sexes (Littell et al. 2006). In other models, the model assuming equal variance between sexes fitted the data best. The residuals of all these models were normally (symmetrically) distributed, judged by visual inspection.

Potential sex and age differences in offspring and parental digit ratios in the correlative data from 2005 were examined using general linear mixed models, where age (fledgling or adult) and sex and their interaction were fitted as a fixed factors and nest of origin as a random factor to control for the resemblance of digit ratios between family members (Littell et al. 2006). Assumption of heterogeneous residual variance by age and sex was examined using AIC, and the residual variance structure having the lowest AIC value was chosen (Littell et al. 2006). In all models except the model for 2D:4D, the model assuming equal residual variance by age and sex fitted the data best. In the case of $2 \mathrm{D}: 4 \mathrm{D}$, the model assuming unequal residual variance by sex and age fitted the data best. The residuals of all these models were normally distributed, judged by visual inspection.

An association between maternal digit ratios and laying date (mean $\pm \mathrm{SD}=27.1 \pm 3.1,1=1.5 .2005)$ as well as the relations of paternal digit ratios with arrival date (mean \pm $\mathrm{SD}=10.4 \pm 3.6,1=1.5 .2005)$, forehead patch size (mean \pm $\mathrm{SD}=17.9 \pm 6.5 \mathrm{~mm}^{2}$ ) and UV chroma of tertials (mean proportion of reflectance in the UV spectrum $\pm \mathrm{SD}=0.23 \pm$ 0.01 ) were studied with Spearman correlation coefficients. 
Due to the categorical nature of clutch size (mean $\pm \mathrm{SD}=6.3 \pm$ 0.6 , range 5-7), an association between maternal digit ratios and clutch size, controlling for laying date, was examined using multinominal logistic regression. Due to high collinearity of digit ratios, separate analyses were conducted for each digit ratio. In multinominal regression, the assumption of proportional odds was confirmed with chi-square score test $\left(\chi^{2}<2.64, P>0.27\right)$.

For the androgen manipulation experiment, general linear mixed models were used, where the response variable were the digit ratios (2D:4D, 2D:3D or 3D:4D) and explanatory variables were androgen treatment, sex and their interaction. Nest of origin and rearing aviary were included as random factors to control for within-brood resemblance of digit ratios and potential effects of the common rearing environment. The residuals of all these models were normally distributed, judged by visual inspection.

The association between digit ratios and activity in the novel object experiment was analysed with a general linear mixed model where the explanatory variables were digit ratios, androgen treatment, sex and treatment-by-sex interaction (as these covariates have been found to affect the tested behavioral traits, see Ruuskanen and Laaksonen 2010). Rearing aviary was included as a random factor. Latency to approach the novel object did not fit any general distribution (and could not be successfully transformed to normality). It was therefore divided into two categories (did/did not approach the object within $3 \mathrm{~min}$ ) and analysed with a logistic regression model, using similar explanatory variables as above. Activity in the novel environment was analysed using a negative binomial distribution with explanatory variables as above. Each digit ratio was tested in a separate model due to collinearity (keeping the explanatory variables the same).

In all mixed models, likelihood ratio test and $F$ test were used to determine the significance of random and fixed terms, respectively (Bolker et al. 2009), and the KenwardRoger method was used for calculating the degrees of freedom of fixed effects (Littell et al. 2006). All analyses were conducted with SAS statistical software version 9.2 (SAS Institute Inc, Cary, NC, USA).

\section{Results}

The digit ratios of fledglings were not associated with yolk testosterone or androstenedione concentration in the correlative data from 2005 (Table 1). In the fledgling model, brood identity explained significant proportion of the variance of digit ratios (Table 1). The experimental elevation of yolk androgen levels did not affect digit ratios either (Table 2).

In the correlative data, 2D:4D did not differ between the sexes in either fledglings or adults (Table 3, Fig. 1a). However, for 2D:3D and 3D:4D, we found an interaction between sex and age (Table 3): There were no differences in digit ratios between males and females in fledglings, but in adults, 2D:3D was higher in males than in females, whereas in 3D:4D, the pattern was the opposite (Fig. 1b, c). These differences were rather due to a difference in the lengths of $2 \mathrm{D}$ and $4 \mathrm{D}$ than in $3 \mathrm{D}$ (mean $\pm \mathrm{SD}, \mathrm{mm}: 2 \mathrm{D}$,
Table 1 The association between yolk androgens, as measured from one egg in the clutch, and digit ratios of fledgling pied flycatchers

In all models, a random factor of nest of origin explained significantly the variance in digit ratios (likelihood ratio test: in all cases $\chi^{2}>2.70, p<0.05$ )

\begin{tabular}{|c|c|c|c|c|}
\hline Digit ratio & $\beta(95 \% \mathrm{CIs})$ & $d f_{\text {num,den }}$ & $F$ & $p$ value \\
\hline \multicolumn{5}{|l|}{ 2D:4D $(N=235)$} \\
\hline Testosterone & $-0.0002(-0.0019,0.0015)$ & $1,67.9$ & 0.28 & 0.60 \\
\hline Androstenedione & $-0.00003(-0.0002,0.0002)$ & $1,72.9$ & 0.00 & 0.95 \\
\hline Sex & & 1,206 & 0.10 & 0.75 \\
\hline Sex $\times$ testosterone & & $1,197.2$ & 0.13 & 0.72 \\
\hline Sex $\times$ androstenedione & & $1,196.6$ & 0.21 & 0.64 \\
\hline \multicolumn{5}{|l|}{$2 \mathrm{D}: 3 \mathrm{D}(N=218)$} \\
\hline Testosterone & $0.00002(-0.00099,0.00093)$ & $1,46.0$ & 0.00 & 0.98 \\
\hline Androstenedione & $0.00001(-0.00010,0.00012)$ & $1,54.9$ & 0.03 & 0.86 \\
\hline Sex & & $1,208.7$ & 0.11 & 0.74 \\
\hline Sex $\times$ testosterone & & $1,192.9$ & 0.00 & 0.98 \\
\hline Sex $\times$ androstenedione & & $1,203.2$ & 0.02 & 0.88 \\
\hline \multicolumn{5}{|l|}{ 3D:4D $(N=218)$} \\
\hline Testosterone & $-0.0005(-0.0030,0.0020)$ & $1,65.08$ & 0.46 & 0.50 \\
\hline Androstenedione & $-0.00003(-0.00032,0.00027)$ & $1,72.5$ & 0.00 & 0.97 \\
\hline Sex & & $1,197.5$ & 0.00 & 0.99 \\
\hline Sex $\times$ testosterone & & $1,185.4$ & 0.11 & 0.74 \\
\hline Sex $\times$ androstenedione & & $1,193.2$ & 0.07 & 0.79 \\
\hline
\end{tabular}


Table 2 The effects of sex and androgen treatment on the digit ratios measured in the androgen manipulation experiment $(N=58)$

\begin{tabular}{llll}
\hline Digit ratio & $d f_{\text {num, den }}$ & $F$ & $p$ value \\
\hline 2D:4D & & & \\
Sex & $1,30.1$ & 2.25 & 0.14 \\
Treatment & $1,27.3$ & 0.10 & 0.75 \\
Sex $\times$ treatment & $1,30.1$ & 0.27 & 0.61 \\
2D:3D & & & \\
Sex & $1,29.9$ & 0.27 & 0.60 \\
Treatment & $1,28.4$ & 0.54 & 0.47 \\
Sex $\times$ treatment & $1,29.9$ & 0.98 & 0.33 \\
3D:4D & $1,28.6$ & 1.46 & 0.24 \\
Sex & $1,27.2$ & 1.22 & 0.28 \\
Treatment & $1,28.6$ & 0.35 & 0.56 \\
Sex $\times$ treatment & & & \\
\hline
\end{tabular}

Nest of origin and rearing conditions (aviary) were included as random factors in all models (likelihood ratio tests in all cases: $\chi^{2}<$ $1.4, p>0.24)$

males, $6.50 \pm 0.31, N=68$; females, $6.35 \pm 0.32, N=77$, $F_{1,143}=8.40, p=0.004 ; 3 \mathrm{D}$, males, $11.00 \pm 0.46, N=67$; females, $10.94 \pm 0.45, N=77, F_{1,142}=0.47, p=0.49 ; 4 \mathrm{D}$, males, $7.37 \pm 0.32, N=68$; females; 7.22 $\pm 0.36, N=77$, $\left.F_{1,143}=6.26, p=0.014\right)$. Furthermore, there was an overall difference in fledgling and adult digit ratios in the correlative data, fledgling 2D:4D and 2D:3D being higher than that of adults, and fledgling 3D:4D being lower than that of adults (Table 3, Fig. 1a-c). No sex differences were found in the digit ratios of the captive adult birds from the androgen manipulation experiment (Table 2).

Table 3 The effects of sex and age (fledgling or adult) on the digit ratios measured in the correlative field data

\begin{tabular}{llll}
\hline Digit ratio & $\boldsymbol{d} \boldsymbol{f}_{\text {num, den }}$ & $\boldsymbol{F}$ & $\boldsymbol{p}$ value \\
\hline 2D:4D $(N=380)$ & & & \\
Sex & 1,239 & 0.00 & 0.99 \\
Age & $1,244.4$ & 13.95 & 0.0002 \\
Sex $\times$ age & $1,235.1$ & 0.68 & 0.41 \\
2D:3D $(N=362)$ & & & \\
Sex & $1,311.8$ & 5.82 & 0.016 \\
Age & $1,325.1$ & 93.17 & $<0.0001$ \\
Sex $\times$ age & $1,304.3$ & 5.07 & 0.025 \\
3D:4D $(N=362)$ & & & \\
Sex & $1,318.5$ & 5.61 & 0.019 \\
Age & $1,329.5$ & 36.96 & $<0.0001$ \\
Sex $\times$ age & 1,313 & 3.52 & 0.062 \\
\hline
\end{tabular}

In all models, a random factor of nest of origin explained significantly the variance of digit ratios (likelihood ratio test: in all cases $\chi^{2}>11.7$, $p<0.0003$ )
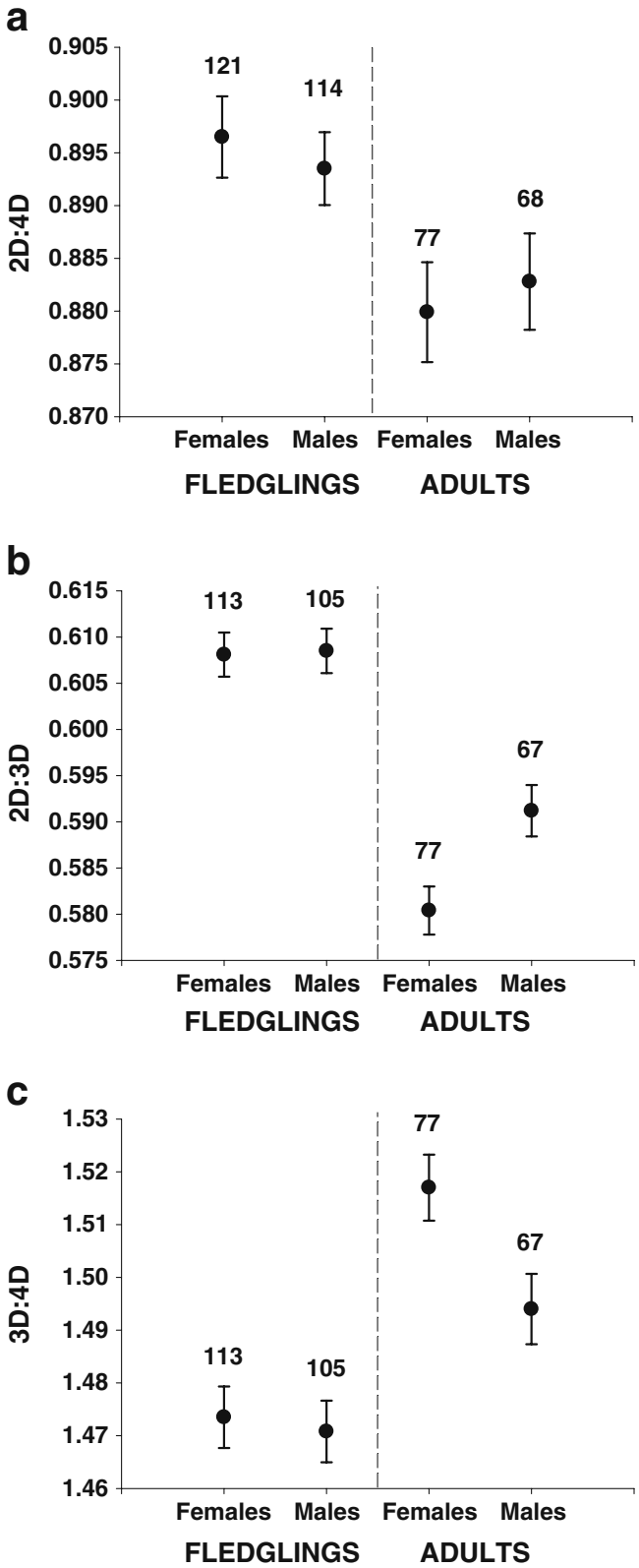

Fig. 1 Estimated marginal means $( \pm \mathrm{SE})$ of digit ratios for fledglings and adults by sex in a 2D:4D, b 2D:3D and $\mathbf{c} 3 \mathrm{D}: 4 \mathrm{D}$. Numbers above the bars represent sample sizes

We found no association between digit ratios and reproductive traits/quality (laying date or clutch size in females, arrival date in males) in either females or males (Table 4). There was further no association between digit ratios and male plumage traits (forehead patch size and UV chroma) (Table 4). Finally, digit ratios were not associated with any of the behavioral traits tested (activity in the presence of a novel object, latency to approach novel object and activity in the novel environment); only a tendency for a negative association between 2D:4D and activity in the novel environment was found (Table 5). 
Table 4 The association between female and male digit ratios and reproductive or plumage traits in the correlative field data

\begin{tabular}{lllll}
\hline & Odds ratio $(95 \% \mathrm{Cls}) / r$ & $n$ & $\chi^{2}$ & $p$ value \\
\hline Males & & & & \\
Arrival date & & & & \\
2D:4D & -0.070 & 66 & & 0.58 \\
2D:3D & 0.060 & 65 & & 0.63 \\
3D:4D & -0.036 & 65 & & 0.78 \\
Patch size & & & & \\
2D:4D & 0.064 & 66 & & 0.61 \\
2D:3D & 0.025 & 65 & & 0.84 \\
3D:4D & -0.003 & 65 & & 0.98 \\
UV chroma & & & & \\
2D:4D & 0.004 & 64 & & 0.98 \\
2D:3D & 0.096 & 63 & & 0.45 \\
3D:4D & -0.095 & 63 & & 0.46 \\
Females & & & & \\
Laying date & & & & \\
2D:4D & -0.075 & 77 & & 0.52 \\
2D:3D & -0.125 & 77 & & 0.28 \\
3D:4D & -0.015 & 77 & & 0.90 \\
Clutch size & & 77 & 0.75 & 0.79 \\
2D:4D & $1.06(0.68,1.65)$ & & 0.79 \\
2D:3D & $0.92(0.59,1.43)$ & 77 & \\
3D:4D & $1.22(0.78,1.89)$ & & & \\
\hline
\end{tabular}

Spearman correlations were calculated between digit ratios and female laying date, male arrival date, patch size and UV chroma. For clutch size, the results are from multinomial regression models (see Methods).

\section{Discussion}

We found no evidence that digit ratios were related to maternally derived yolk androgen exposure in the pied flycatcher. Experimental elevation of yolk androgen level had no effect on digit ratios in adults, and there was no correlation between yolk androgen level measured from one egg in a clutch (a good proxy of the clutch mean) and digit ratios of the remaining fledglings. Furthermore, we found no associations between digit ratios and either measures of reproduction in the wild or behaviour in standardised laboratory tests. These results thus indicate that digit ratios are not reliable markers of maternally derived early androgen exposure and that they should be used with caution as phenotypic markers of evolutionary importance. There was, however, a sex difference in adult birds in the wild and the digit ratios of adult birds differed from those of fledglings. This may suggest that the sex difference in digit ratio develops at a later stage of development than at fledging (when the chicks are otherwise already fully grown).

Digit ratios and early androgen exposure

We did not find any effect of maternally derived androgen levels on digit ratios. However, considering the lack of correlation between fledgling digit ratios and yolk androgen level, one must bear in mind that if the digit ratios of fledglings are not yet fully developed, the result on the effects of yolk androgens on them should be interpreted with caution. Furthermore, we used a rather high level of elevation of yolk androgens, which may have resulted in androgen levels higher than the physiological range for some individuals (see Methods).

Our results contrast the findings of the few previous experimental studies in birds that have shown some evidence for digit ratios reflecting maternally derived hormone levels: High androgen exposure in the yolk led to higher 2D:3D (but not 2D:4D or 3D:4D) in female pheasants, which was due to shorter 3D in individuals hatching from androgen-elevated eggs (Romano et al. 2005), whereas the elevation of estradiol levels in egg
Table 5 The association between digit ratios and behavioral traits in the androgen manipulation experiment

Note that each digit ratio was tested in a separate model due to collinearity. In all models, sex, androgen treatment and their interaction were controlled for

\begin{tabular}{|c|c|c|c|c|}
\hline & $\beta(95 \% \mathrm{Cls})$ & $d f_{\text {num,den }}$ & $F$ & $p$ value \\
\hline \multicolumn{5}{|c|}{ Activity in the novel object experiment } \\
\hline 2D:4D & $2.24(-282.26,286.75)$ & $1,49.4$ & $<0.01$ & 0.99 \\
\hline $2 \mathrm{D}: 3 \mathrm{D}$ & $-77.07(-560.97,406.83)$ & $1,51.8$ & 0.1 & 0.75 \\
\hline $3 \mathrm{D}: 4 \mathrm{D}$ & $26.7(-162.46,215.80)$ & $1,47.8$ & 0.08 & 0.78 \\
\hline \multicolumn{5}{|c|}{ Latency to approach the novel object } \\
\hline 2D:4D & $-5.18(-26.63,16.25)$ & 1,52 & 0.24 & 0.63 \\
\hline $2 \mathrm{D}: 3 \mathrm{D}$ & $-9.28(-46.81,28.25)$ & 1,52 & 0.25 & 0.62 \\
\hline 3D:4D & $-1.61(-16.08,12.84)$ & 1,52 & 0.05 & 0.82 \\
\hline \multicolumn{5}{|c|}{ Activity in the novel environment } \\
\hline 2D:4D & $-13.24(-26.83,0.34)$ & 1,53 & 3.82 & 0.06 \\
\hline $2 \mathrm{D}: 3 \mathrm{D}$ & $-9.80(-33.41,13.82)$ & $1,52.99$ & 0.69 & 0.41 \\
\hline 3D:4D & $-4.24(-13.45,5.16)$ & 1,53 & 0.82 & 0.37 \\
\hline
\end{tabular}


yolks of pheasants decreased 2D:4D in males only (Saino et al. 2007). When compared to the control groups, it appears that testosterone treatment masculinised the 2D:3D of females while estradiol treatment feminised the 2D:4D of males. Both of these experimental studies were conducted with captive strains of pheasants, and our contrasting results in a wild bird species thus question the generality of these prior findings until there is more evidence from other species.

All in all, the development of digits seems to be a more complex process than previously generally believed. In addition to hormonal effects, a large additive genetic component for variation in digit ratios has been found for both mammals and birds (Ramesh and Murty 1977; Forstmeier 2005; Paul et al. 2006; Voracek and Dressler 2009). In line with this, we found significant among-brood variation in digit ratios, which may indicate a genetic component (but also unmeasured maternal or environmental components) of digit ratios. However, the proximate factors determining digit ratios may even vary among populations, for example due to genetic bottlenecks (e.g. Manning et al. 2000; Forstmeier 2005).

It should be noted that our study and the abovementioned studies on birds have investigated the effect of maternally derived steroids on digit ratio development and other phenotypic and behavioral correlates. The origin of the hormones affecting digit ratios and other traits is however unclear, as either maternal hormones (or maternally derived yolk hormones in egg-laying animals) or hormones produced by the embryo itself (or by the foetal part of the placenta in some mammal species) could directly affect both digit ratio and development of other traits. Another possibility is that Hox genes may have pleiotropic effects on the development of digits and the gonads, the latter of which influences the embryonic production of sex hormones. Furthermore, steroid hormones could even affect the expression of these genes (see Forstmeier et al. 2008 for a detailed review). Evidence for or against any of the abovementioned mechanisms is still quite sparse, but in mammals, it is often presumed that mainly steroids produced by the embryo, not the mother, have pleiotropic effects on digit development and other traits (e.g. reviewed by Manning et al. 2000; Manning 2002, 2008; McIntyre 2006). In egg-laying animals, the embryo itself produces steroids during a period (for example in chickens from day 7 onwards, Rogers 1995), which overlaps with the period of digit development. The doses of steroids from the developing gonads inducing sexual differentiation are larger than those of maternal origin (at least 2-3-fold, reviewed by Carere and Balthazart 2007). This suggests that the role of steroids of embryonic origin in digit development may be more important than of those of maternal origin also in birds. Furthermore, the effects of manipulation of mater- nally derived androgen or oestrogen levels (Romano et al. 2005; Saino et al. 2007, see above) on digit ratios could also be manifested via organising effects on embryo's own androgen production and not directly on digits (e.g. priming of the phalangeal anlagen, Forstmeier et al. 2008). Therefore, the role of steroids of embryonic vs. maternal origin on digit ratios in birds should be further examined.

In addition, the relative contribution of different steroids such as estrogens on digit ratio determination on different taxa should also be considered. A recent study on zebra finches suggests that estrogens, not androgens, are associated with the development of digit ratios and may explain co-variation between digit ratios and other traits (Forstmeier et al. 2010). The lack of testosterone dependence of digit ratios found in this study does not exclude this possibility.

\section{Sex difference in adult digit ratios}

The sex differences found in the digit ratios of adult birds were not yet apparent in fledgling birds or in the captive birds several months after fledging while reaching full independence. This may indicate that digits further develop long after fledging, and thus, gonadal sex hormones most likely affect digit ratio development. Experimental manipulation of hormone (oestrogen) levels during main growth period (nestling phase) did not affect digit ratios (Forstmeier et al. 2008). We may interpret this as suggesting that hormonal levels at later phases may be more important for digit ratio determination. Sex hormones have been shown to greatly affect digit growth during puberty in mammals (reviewed in Perry et al. 2008), but it is uncertain when the actual digit ratios are determined and how stable these are (see, e.g. Manning 2002; McIntyre et al. 2005; McIntyre 2006; Gillam et al. 2008). The digit ratios of our captive birds were measured in the autumn, before the growth of the reproductive organs at the step of the first breeding season in the following spring (i.e. the time which could correspond to puberty, as sex hormone levels are high). We therefore suggest that the sex difference in breeding adult birds may be explained by a difference arising during reproductive maturation. This would be in line with the finding that in human females digit ratios might vary with respect to hormonal state (menstrual cycle) during adulthood (Mayhew et al. 2007). Interestingly, Garamszegi et al. (2008) also found that digit ratios of juvenile birds (1-year-old) differed from those of older individuals in male collared flycatchers, which may also support this idea. An alternative explanation for the sex difference in adults from the wild may lie in selection, assuming that digit ratios are associated with fitness-related traits (but see below). Furthermore, agedependent differences in the volume of soft tissues (possibly differing among sexes and digits) may potentially also 
explain some of the variation in the age-dependent results found here.

Sex differences in digit ratios have been recorded in many vertebrate species (e.g. Manning et al. 1998, 2002; Brown et al. 2002; Roney et al. 2004; Rubolini et al. 2006), but the direction of the difference seems to vary between taxa. In mammals, males seem to have lower 2D:4D ratios than females (Manning et al. 1998, 2002; Putz et al. 2004), while in birds and reptiles, the pattern (when found) seems to be the opposite (Burley and Foster 2004; Rubolini et al. 2006; Navarro et al. 2007; Saino et al. 2007; Leoni et al. 2008; this study; but see Chang et al. 2006). However, the picture for birds remains unclear, as a number of studies have not found sexually dimorphic digit ratios (Forstmeier 2005; Romano et al. 2005; Dreiss et al. 2008; Lombardo et al. 2008). The mixed results of sex dimorphism in digit ratios among birds may be due to differences in sensitivity of digit development to sex steroids or for example differences in selection between sexes for a certain foot shape, as suggested by Romano et al. (2005) and Lombardo et al. (2008).

Are digit ratios phenotypic markers for traits of evolutionary importance?

Regardless of the exact mechanism of digit ratio determination, an interesting question is whether digit ratios have any clear indicator value in an evolutionary context, as this would facilitate the laborious study of fitness effects and adaptations. However, we did not find any associations of digit ratios with female or male reproductive traits (laying date and clutch size in females, arrival date in males). There are only few studies on the associations between digit ratios and any traits related to reproductive success or fertility, and those have also shown mixed results (for humans, reviewed by Putz et al. 2004).

Furthermore, we did not find any correlations between digit ratios and male secondary sexual plumage traits (forehead patch size and UV chroma, e.g. Lundberg and Alatalo 1992; Siitari et al. 2002). This is parallel to findings in the collared flycatcher, a sister species to the pied flycatcher, in which digit ratios were not associated with any song traits or forehead or wing patch sizes (Garamszegi et al. 2008). Digit ratios were not related to male attractiveness in zebra finches either, although there was an association between digit ratios and male song rate (Forstmeier 2005). Two sexually dimorphic traits, tail length in swallows and badge size in house sparrows were found to be associated with digit ratios (Navarro et al. 2007; Dreiss et al. 2008), but the results could not be replicated in another study (Lombardo et al. 2008). This implies a complex and rather weak relationship of digit ratios with plumage traits in birds.
Finally, we did not find association between digit ratios and the behavioral traits tested (exploration of a novel environment, activity and neophobia). The behaviours we measured were not specifically sexual behaviours, which may have reduced the likelihood of finding correlations with digit ratios. Most of the studies on humans and other vertebrates in which correlations between digit ratios and behavioral traits have been found have investigated specifically sex-related behaviours and phenotypic traits (e.g. sexual orientation, cognitive abilities, social dominance, appearance and vigour; reviewed by Putz et al. 2004). These are presumed to be more affected by early androgen exposure than traits that are not sex-specific. To put together, our results strongly suggest that digit ratios cannot be used as phenotypic indicators for fitness-related or behavioral traits, at least in our study species.

\section{Conclusions}

Our results indicate that digit ratios are not associated with maternally derived yolk androgens in pied flycatchers, thus downplaying their importance as a general phenotypic marker for maternally derived early androgen exposure. We nevertheless found evidence for sex differences in digit ratios (2D:3D and 3D:4D) in adult birds, and we suggest that hormones during sexual maturation for breeding may play a role in digit ratio determination in this species. Furthermore, digit ratios were not associated with any of the reproductive traits, male secondary sexual traits or behavioral traits studied, suggesting that digit ratios may bear little relevance as phenotypic indicators of these adaptive traits.

Acknowledgements We thank Jorma Nurmi and Esa Lehikoinen for their help in field monitoring and Academy of Finland (grant to TL and grant no. 207270 to SH), Emil Aaltonen Foundation (grant to TL) and Finnish Cultural Foundation (grant to SR) for funding.

Open Access This article is distributed under the terms of the Creative Commons Attribution Noncommercial License which permits any noncommercial use, distribution, and reproduction in any medium, provided the original author(s) and source are credited.

\section{References}

Allaway HC, Bloski TG, Pierson RA, Lujan ME (2009) Digit ratios (2D:4D) determined by computer-assisted analysis are more reliable than those using physical measurements, photocopies, and printed scans. Am J Hum Biol 21:365-370

Bertin A, Richard-Yris MA, Houdelier C, Lumineau S, Möstl E, Kuchar A, Hirschenhauser K, Kotrschal K (2008) Habituation to humans affects yolk steroid levels and offspring phenotype in quail. Horm Behav 54:396-402 
Bolker BM, Brooks ME, Clark CJ, Geange SW, Poulsen JR, Stevens MHH, White J-S (2009) Generalized linear mixed models: a practical guide for ecology and evolution. Trends Ecol Evol 24:127-135

Brown WM, Finn CJ, Breedlove SM (2002) Sexual dimorphism in digit-length ratios of laboratory mice. Anat Rec 267:231-234

Burley NT, Foster VS (2004) Digit ratio varies with sex, egg order and strength of mate preference in zebra finches. Proc R Soc Lond B 271:239-244

Carere C, Balthazart J (2007) Sexual versus individual differentiation: the controversial role of avian maternal hormones. Trends Endocrinol Metab 18:73-80

Chang JL, Doughty S, Wade J, Lovern MB (2006) Sexual dimorphism in the second-to-fourth digit length ratio in green anoles, Anolis carolinensis (Squamata: Polychrotidae), from the southeastern United States. Can J Zool 84:1489-1494

Clark MM, Galef BG Jr (1995) Prenatal influences on reproductive life history strategies. Trends Ecol Evol 10:151-153

Cohen-Bendahan CCC, van de Beek C, Berenbaum SA (2005) Prenatal sex hormone effects on child and adult sex-typed behavior: methods and findings. Neurosci Biobehav Rev 29:353-384

Dreiss AN, Navarro C, de Lope F, Møller AP (2008) Digit ratios, secondary sexual characters and condition in barn swallows Hirundo rustica. Behav Ecol 19:16-21

Dufty AM, Clobert J, Møller AP (2002) Hormones, developmental plasticity and adaptation. Trends Ecol Evol 17:190-196

Eaton MD, Lanyon SM (2003) The ubiquity of avian ultraviolet plumage reflectance. Proc R Soc Lond B 270:1721-1726

Forstmeier W (2005) Quantitative genetics and behavioral correlates of digit ratio in the zebra finch. Proc R Soc Lond B 272:26412649

Forstmeier W, Rochester J, Millam JR (2008) Digit ratio unaffected by estradiol treatment of zebra finch nestlings. Gen Comp Endocrinol 156:379-384

Forstmeier W, Mueller JC, Kempenaers B (2010) A polymorphism in the oestrogen receptor gene explains covariance between digit ratio and mating behavior. Proc R Soc Lond B 277:3353-3361

Garamszegi LZ, Hegyi G, Szöllosi E, Rosivall B, Török J, Eens M, Møller AP (2008) Phenotypic correlates of digit ratio in a wild bird: implications for the study of maternal effects. Anim Behav 74:641-647

Gillam L, McDonald R, Ebling FJP, Mayhem TM (2008) Human 2D (index) and 4D (ring) finger lengths and ratios: cross-sectional data on linear growth patterns, sexual dimorphism and lateral asymmetry from 4 to 60 years of age. J Anat 213:325-335

Groothuis TGG, Schwabl H (2002) Determinants of within- and among-clutch variation in levels of maternal hormones in blackheaded gull eggs. Funct Ecol 16:281-289

Groothuis TGG, von Engelhardt N (2005) Investigating maternal hormones in avian eggs: measurement, manipulation, and interpretation. Ann NY Acad Sci 1046:168-18

Groothuis TGG, Müller W, von Engelhardt N, Carere C, Eising CM (2005) Maternal hormones as a tool to adjust offspring phenotype in avian species. Neurosci Biobehav Rev 29:329-352

Hackl R, Bromundt V, Daisley J, Kotrschal K, Möstl E (2003) Distribution and origin of steroid hormones in the yolk of Japanese quail eggs (Coturnix coturnix japonica). J Comp Physiol B 173:327-331

Hirschenhauser K, Möstl E, Kotrschal K (1999) Seasonal patterns of sex steroids determined from feces in different social categories of Greylag geese (Anser anser). Gen Comp Endocrinol 114:67-79

Hönekopp J, Bartholdt L, Beier L, Liebert A (2007) Second to fourth digit length ratio (2D:4D) and adult sex hormone levels: new data and a meta-analytic review. Psychoneuroendocrinology 32:313321
Laaksonen T, Lessells K (2006) Maternal effects as mediators of sexual conflict over parental care. J Ornithol 47:11

Laaksonen T, Adamczyck F, Ahola MP, Möstl E, Lessells CM (2010) Yolk androgens and sexual conflict in the pied flycatcher. Behav Ecol Sociobiol, in press

Lehtonen P, Primmer CR, Laaksonen T (2009) Different traits affect gain of extrapair paternity and loss of paternity in the pied flycatcher, Ficedula hypoleuca. Anim Behav 77:1103-1110

Leoni B, Rubolini D, Romano M, di Giancamillo M, Saino N (2008) Avian hind-limb digit length ratios measured from radiographs are sexually dimorphic. J Anat 213:425-430

Littell RC, Milliken GA, Stroup WW, Wolfinger RD (2006) SAS system for mixed models. SAS Institute Inc., Cary

Lombardo MP, Thorpe PA, Brown BM, Sian K (2008) Digit ratio in birds. Anat Rec 291:1611-1618

Lundberg A, Alatalo R (1992) The pied flycatcher. Poyser, London

Manning JT (2002) Digit ratio. Rutgers University Press, New Brunswick

Manning JT (2008) The finger book. Faber and Faber Limited, London

Manning JT, Scutt D, Wilson J, Lewis-Jones DI (1998) The ratio of 2nd to 4th digit length: a predictor of sperm numbers and concentrations of testosterone, luteinizing hormone and oestrogen. Human Repr 13:3000-3004

Manning JT, Barley L, Walton J, Lewis-Jones DI, Trivers RL, Singh D, Thornhill R, Rohde P, Bereczkei T, Henzi P, Soler M, Szwed A (2000) The 2nd:4th digit ratio, sexual dimorphism, population differences, and reproductive success: evidence for sexually antagonistic genes? Evol Hum Behav 21:163-183

Mayhew TM, Gillam L, McDonald R, Ebling FJP (2007) Human 2D (index) and 4D (ring) digit lengths: their variation and relationships during the menstrual cycle. J Anat 211:630-638

McIntyre MH (2006) The use of digit ratios as markers for perinatal androgen action. Reprod Biol Endocrinol 4:10

McIntyre MH, Ellison PT, Lieberman DE, Demerath E, Towne B (2005) The development of sex differences in digital formula from infancy in the fels longitudinal study. Proc R Soc Lond B 272:1473-1479

Michl G, Török J, Péczely P, Garamszegi LZ, Schwabl H (2005) Female collared flycatchers adjust yolk testosterone to male age, but not to attractiveness. Behav Ecol 16:383-388

Möstl E, Spendier H, Kotrschal K (2001) Concentration of immunoreactive progesterone and androgens in the yolk of hen's egg (Gallus domesticus). Wiener Tierärztl Monatsschr $88: 62-65$

Navarro C, de Lope F, Møller AP (2007) Digit ratios (2D:4D), secondary sexual characters and cell-mediated immunity in house sparrows Passer domesticus. Behav Ecol Sociobiol 61:11611168

Palme R, Möstl E (1994) Biotin-streptavidin enzyme immunoassay for the determination of oestrogens and androgens in boar faeces. In: Görög S (ed) Advances of steroid analysis '93. Akadémiai Kiadó, Budapest, pp 111-117

Paul SN, Kato BS, Cherkas LF, Andrew T, Spector TD (2006) Heritability of the second to fourth digit ratio (2D 4D): a twin study. Twin Res Hum Genet 9:215-219

Perry RJ, Farquharson C, Ahmed SF (2008) The role of sex steroids in controlling pubertal growth. Clin Endocrinol 68:4-15

Pilz KM, Quiroga M, Schwabl H, Adkins-Regan E (2004) European starling chicks benefit from high yolk testosterone levels during a drought year. Horm Behav 46:179-192

Putz DA, Gaulin SJC, Sporter RJ, McBurney DH (2004) Sex hormones and finger length: what does 2D:4D indicate? Evol Hum Behav 25:182-199

Ramesh A, Murty JS (1977) Variation and inheritance of relative length of index finger in man. Ann Hum Biol 4:479-484 
Reed WL, Vleck CM (2001) Functional significance of variation in egg-yolk androgens in the American coot. Oecologia 128: $164-171$

Rogers LJ (1995) The development of brain and behaviour in the chicken. $\mathrm{CAB}$ International, the University Press, Cambridge

Romano M, Rubolini D, Martinelli R, Alquati AB, Saino N (2005) Experimental manipulation of yolk testosterone affects digit length ratios in the ring-necked pheasant (Phasianus colchicus). Horm Behav 48:342-346

Roney JR, Whitham JC, Leoni M, Bellem A, Wielebnowski N, Maestripieri D (2004) Relative digit lengths and testosterone levels in Guinea baboons. Horm Behav 45:285-290

Rubolini D, Pupin F, Sacchi R, Gentilli A, Zuffi MAL, Galeotti P, Saino N (2006) Sexual dimorphism in digit length ratios in two lizard species. Anat Rec 288A:491-497

Ruuskanen S, Laaksonen T (2010) Yolk hormones have sex-specific long-term effects on behavior in the pied flycatcher (Ficedula hypoleuca). Horm Behav 57:119-127

Ruuskanen S, Doligez B, Tschirren B, Pitala N, Gustafsson L, Groothuis TGG, Laaksonen T (2009) Yolk androgens do not appear to mediate sexual conflict over parental investment in the collared flycatcher Ficedula albicollis. Horm Behav 55:514-519

Saino N, Rubolini D, Romano M, Boncoraglio G (2007) Increased egg estradiol concentration feminizes digit ratios of male pheasants (Phasianus colchicus). Naturwissenschaften 94:207212

Siitari H, Honkavaara J, Huhta E, Viitala J (2002) Ultraviolet reflection and female mate choice in the pied flycatcher, Ficedula hypoleuca. Anim Behav 63:97-102

Talarovičová A, Kršková L, Blažeková J (2009) Testosterone enhancement during pregnancy influences the 2D:4D ratio and open field motor activity of rat siblings in adulthood. Horm Behav 55:235-239

Tobler M, Granblom M, Sandell M (2007) Maternal androgens in the pied flycatcher: timing of breeding and within-female consistency. Oecologia 151:731-760

Tschirren B, Richner H, Schwabl H (2004) Ectoparasite-modulated deposition of maternal androgens in great tit eggs. Proc R Soc Lond B 271:1371-1375

Tschirren B, Saladin V, Fitze PS, Schwabl H, Richner H (2005) Maternal yolk testosterone does not modulate parasite susceptibility or immune function in great tit nestlings. J Anim Ecol 74:675-682

Verbeek MEM, Drent PJ, Wiepkema PR (1994) Consistent individual differences in early exploratory behavior of male great tits. Anim Behav 48:1113-1121

Voracek M, Dressler SG (2009) Brief communication: familial resemblance in digit ratio (2D:4D). Am J Phys Anthropol $140: 376-380$ 